\title{
The Cardiac Function in the Beach Chair Position under General Anesthesia
}

\author{
Kumiko Tanabe ${ }^{1 *}$, Yuko Yamada1, Kiyoshi Nagase', Nobuo Terabayashi², Hiroki lida ${ }^{1}$ \\ ${ }^{1}$ Department of Anesthesiology and Pain Medicine, Gifu University Graduate School of Medicine, Gifu, Japan \\ ${ }^{2}$ Department of Orthopaedic Surgery, Gifu University Graduate School of Medicine, Gifu, Japan \\ Email: *kumiko-t@m2.gyao.ne.jp
}

How to cite this paper: Tanabe, K., Yamada, Y., Nagase, K., Terabayashi, N. and Iida, H. (2018) The Cardiac Function in the Beach Chair Position under General Anesthesia. Open Journal of Anesthesiology, 8, 27-34.

https://doi.org/10.4236/ojanes.2018.81003

Received: December 12, 2017

Accepted: January 26, 2018

Published: January 29, 2018

Copyright (๑) 2018 by authors and Scientific Research Publishing Inc. This work is licensed under the Creative Commons Attribution International License (CC BY 4.0).

http://creativecommons.org/licenses/by/4.0/

(c) (i) Open Access

\begin{abstract}
Background: Shoulder surgery is performed in the beach chair position (BCP). The systemic arterial blood pressure (BP) must be increased to prevent cerebral hypoperfusion. However, it is not clear how the cardiac function is affected when BP increase to maintain cerebral perfusion pressure in anesthetized patients. Methods: An analysis was performed using the data from 13 patients. We prepared a parallel circuit using a FloTrac Sensor transducer and an arterial BP transducer. Following the transfer of the patient to the BCP under general anesthesia, the FloTrac Sensor transducer was placed at the level of the fourth intercostal space, the arterial BP transducer was placed at the external auditory meatus level. We selected two points before surgery $(120 \mathrm{~s}$ apart), during which the mean arterial $\mathrm{BP}(\mathrm{mABP})$ at the level of the brain was stable and at which the values in the supine position and the BCP were within $5 \mathrm{mmHg}$. Results: While the patients were in the supine position, the mean $\mathrm{mABP}$ at the mid-axillary level was $65.7 \mathrm{mmHg}$. In the BCP, the mean $\mathrm{mABP}$ was $66.5 \mathrm{mmHg}$ at the external auditory meatus and $80.7 \mathrm{mmHg}$ at the fourth intercostal space. The cardiac index changed from 2.2 (supine position) to $2.5 \mathrm{l} / \mathrm{min} / \mathrm{m}^{2}(\mathrm{BCP})$. The stroke volume index was significantly increased from 35.8 to $42.3 \mathrm{ml} / \mathrm{m}^{2}(P=0.003)$. The heart rate changed from 63.0 to 58.6 beats/min. The stroke volume variation was significantly decreased from $12.4 \%$ to $8.8 \%(P=0.024)$. Conclusion: In order to ensure patient safety, close attention should be paid to the systemic cardiovascular changes that occur when the BP is increased.
\end{abstract}

\section{Keywords}

Beach Chair Position, Cardiac Function, Cerebral Perfusion

\section{Introduction}

Arthroscopic or open shoulder surgeries are performed in the beach chair posi- 
tion (BCP) or the lateral decubitus position [1]. There is no evidence that one position is superior to the other, and both have advantages and disadvantages [1]. Surgical procedures involving a combination of the lateral decubitus position and regional anesthesia are poorly tolerated, and regional anesthesia can be associated with inopportune patient movement during surgery [1]. Regional or general anesthesia may be utilized in conjunction with the BCP [1]; however, under general anesthesia, the BCP is associated with an increased risk of neurological complications, including stroke, spinal cord ischemia, and transient loss of vision [2] [3]. The pathophysiology of these events has not been completely determined but it has been suggested to be related to cerebral or upper spinal cord hypoperfusion due to improper blood pressure (BP) management [2] [3]. Thus, anesthesiologists supply oxygenated blood to the brain through various interventions, including the regulation of the systemic arterial BP or end-tidal carbon dioxide [3] [4] [5].

In a conscious human, postural changes directly influence the cardiac preload and afterload [6]. Tilting the head up from the supine position has an immediate affect on the perfusion of the brain; the grater the difference between the pressure within the heart and the brain, the greater the impact on perfusion of the brain [6]. Thus, when correcting the BP to maintain an adequate cerebral perfusion pressure, one must account for the hydrostatic pressure gradient between the brain and the site at which the BP is measured [6]. However, under normal physiological conditions, the cerebral blood flow is kept at a constant level when the mean arterial $\mathrm{BP}(\mathrm{mABP})$ is between 50 and $150 \mathrm{mmHg}$ [7]. In conscious individuals, there is a significant increase in the $\mathrm{mABP}$ of the upper extremities after a change in posture from the supine position to the BCP [8] [9], while the cerebral tissue oxygen saturation $\left(\mathrm{SctO}_{2}\right)$, a noninvasive indicator of cerebral perfusion, is unaffected by postural change [9] [10]. Moving into an upright position activates the sympathetic nervous system, resulting in an increase in the systemic vascular resistance (SVR) and the systemic BP and a reduction in the cardiac output (CO) [11]. In contrast, the upper extremity $\mathrm{mABP}$ and $\mathrm{SctO}_{2}$ values are significantly decreased when the position of an anesthetized patient is changed to the BCP [8] [9] [10] [12]. It has been suggested that the hydrostatic pressure gradient between the brain and the upper extremities plays a role in the decrease in the brain BP that is observed when anesthetized patients are placed into the $\mathrm{BCP}$ and that the $\mathrm{SctO}_{2}$ value decreases due to cerebral hypoperfusion [8] [9]. Based on this hypothesis, it is recommended that the BP be measured at the brain level or corrected to the brain level from the site at which the BP is measured when the patient is in the BCP [3] [4] [8] [10]. In line with this strategy, the BP at the level of the heart is higher than the BP at the level of the brain. General anesthesia causes a blunting of the baroreceptor responses (leading to an attenuation of the increase in the SVR that is observed when a conscious individual rises from the supine position), while the BP at the level of the heart and the $\mathrm{CO}$ are reduced [11]. However, it is not clear how the cardiac function is af- 
fected when the BP is increased to the level that is required to maintain cerebral perfusion pressure in anesthetized patients in the BCP. Accordingly, we investigated the changes in the cardiac index (CI), and stroke volume index (SVI) that occur when a patient receiving the standard, recommended BP support, is moved to the BCP from a supine position while under general anesthesia.

\section{Materials and Methods}

\subsection{Anesthetic Management}

The present study was approved by Gifu University Graduate School of Medicine Ethics Committee (Gifu, Japan). The study was registered in the University Hospital Medical Information Network in Japan (registration number: UMIN 000017158). Twenty-five consecutive patients enrolled in this study and provided written informed consent. All of the patients refrained from any oral intake or intravenous infusion for $10 \mathrm{~h}$ before the induction of anesthesia. General anesthesia was induced with thiopental and remifentanil and maintained with sevoflurane and remifentanil. The patients were mechanically ventilated at a rate and tidal volume that maintained normocapnia (as measured by end-tidal capnography). The depth of anesthesia was monitored with a Bispectral Index Monitor (BIS) (Medtronic Minimally Invasive Therapies, Minneapolis, MN, USA) and maintained within a BIS target range of 40 to 60 . All of the patients were positioned in a $60^{\circ}$ head-up position (BCP). The patient's BP was managed using standard clinical practices (intravenous ephedrine and phenylephrine, the adjustment of the anesthetic concentration, and the alteration of fluid load), and was measured at the external auditory meatus level with the patient in the BCP.

\subsection{The Measurement of Cardiac Function}

We prepared a parallel circuit using a FloTrac Sensor transducer (Edwards Lifesciences, Irvine, CA, USA) and an arterial BP transducer. Following the induction of anesthesia, a 22-gauge cannula was placed into the non-surgical radial artery and connected to the combination circuit. The patient data were extracted from the FloTrac Sensor using an EV1000 monitor (software version 1.5) (Edwards Lifesciences, Irvine, CA, USA), which estimates the CO, CI, stroke volume (SV), SVI and stroke volume variation (SVV). Both transducers were placed at the mid-axillary level while the patient was in the supine position. When the patient was shifted into the BCP, the FloTrac Sensor transducer was moved to the level of the heart (the fourth intercostal space) for the continuous measurement of the cardiac function and the mABP. The arterial BP transducer was placed at the level of the external auditory meatus for the continuous measurement of the $\mathrm{mABP}$ at the level of the brain.

\subsection{Statistical Analysis}

During the time between the induction of anesthesia and the start of surgery, we selected two points (during $120 \mathrm{~s}$ ) from the anesthetic record at which the mABP 
at the level of the brain was stable (within $5 \mathrm{mmHg}$ ) in both the supine position and the BCP. The paired Student's $t$-test was used to compare the mABP values measured at the level of the heart, as well as CI, SVI, and SVV recorded at these two time points using Excel for Macintosh, version 14.6.0 (Microsoft Redmond, WA, USA). $\mathrm{P}$ values of $<0.05$ were considered to indicate statistical significance. We estimated that 25 patients would be needed because this study protocol was within-subject design, power analysis statistical power as $80 \%$, and based on our previous anesthetic records in which BCP-related $\mathrm{mABP}$ elevation and standard deviation (SD) of $\mathrm{mABP}$ change was 10 and $5 \mathrm{mmHg}$, respectively.

We performed an interim analysis at the half sample size of protocol design using the Pocock method and set the $\mathrm{p}$ value to 0.029 (instead of 0.05 ) to avoid increasing the chance of a type 1 error [13]. The data were expressed as the mean \pm SD.

\section{Results}

From May 2015 to March 2016, 22 consecutive patients undergoing shoulder surgery in the BCP at Gifu University Hospital were enrolled in the present study. Nine patients were excluded because of technical failures from the analysis due to the following factors: hemodynamic instability $(n=4)$, failure of radial artery cannulation $(n=2)$, transducer placement error $(n=2)$, and use of propofol for anesthetic maintenance $(n=1)$. Thus, the data of 13 patients were included in the analysis. The characteristics of the patient are listed in Table 1. The mean distance between the fourth intercostal space and the external auditory meatus was $20.4 \pm 1.8 \mathrm{~cm}$.

Following the induction of anesthesia, while the patients were in the supine position, the $\mathrm{mABP}$ at the mid-axillary level was $65.7 \pm 7.4 \mathrm{mmHg}$. After repositioning the patients in the $\mathrm{BCP}$, the $\mathrm{mABP}$ was $66.5 \pm 8.1 \mathrm{mmHg}$ at the external auditory meatus and $80.7 \pm 8.4 \mathrm{mmHg}$ at the fourth intercostal space (Figure $1(\mathrm{a})$ ). The $\mathrm{mABP}$ values recorded at the level of the heart while the patients were

Table 1. The characteristics of the patients in the present study $(\mathrm{n}=13)$.

\begin{tabular}{cc}
\hline Variables & \\
\hline Age (years) & $64.5 \pm 9.5$ \\
Male/Female & $10 / 3$ \\
Height $(\mathrm{cm})$ & $162.5 \pm 8.5$ \\
Body weight (kg) & $67.5 \pm 8.6$ \\
Distance between two transducers (cm) & $20.4 \pm 1.8$ \\
Hypertension (n) & 6 \\
Diabetes mellitus (n) & 4 \\
Others (n) & CAD (1), CKD (1), HL (1), old Tb (1), RA(1)
\end{tabular}

The values indicate the mean \pm standard deviation. $n$, number of patients; distance between two transducers, distance between the forth intercostal level and the external auditory meatus level in the beach chair position; $\mathrm{CAD}$, coronary artery disease; $\mathrm{CKD}$, chronic kidney disease; HL, hyperlipidemia; $\mathrm{Tb}$, tuberculosis RA, rheumatoid arthritis. 


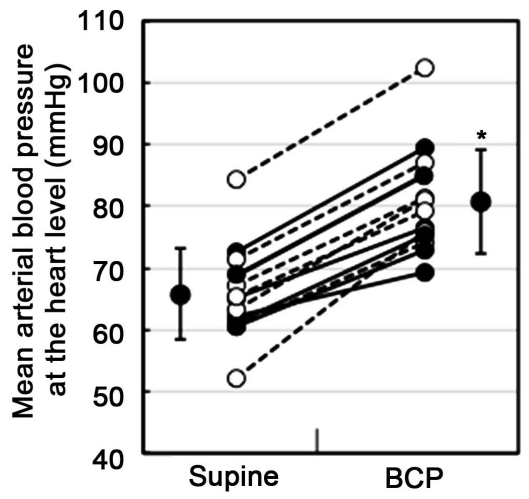

(a)

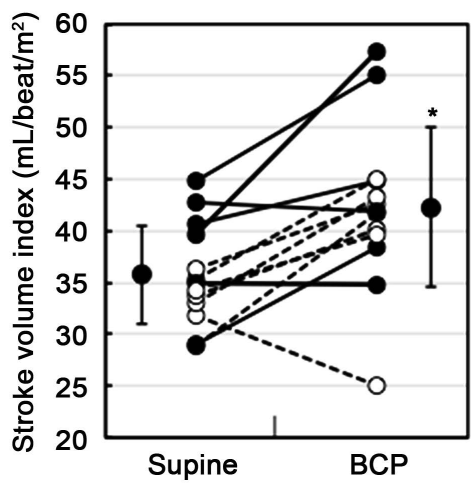

(c)

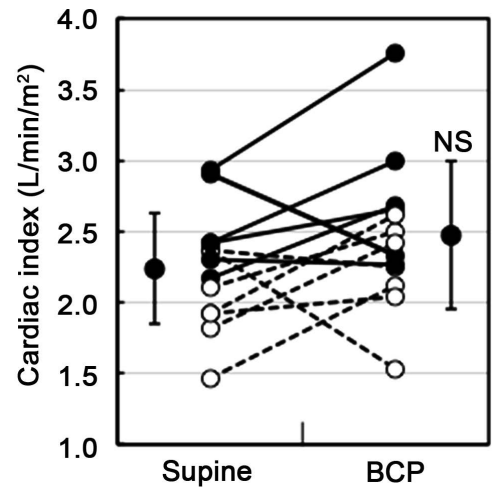

(b)

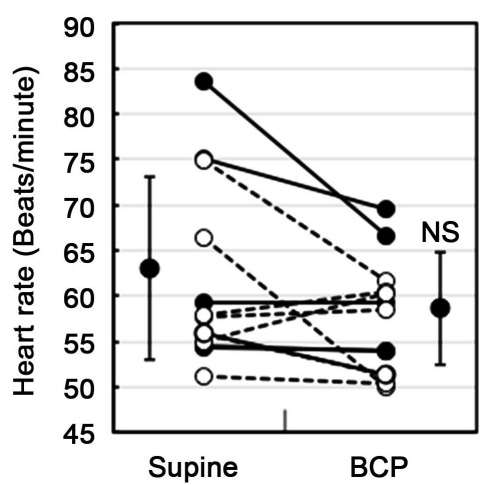

(d)

Figure 1. The hemodynamic values in the supine position and the beach chair position (BCP) in patients receiving blood pressure support. In the BCP, the blood pressure was maintained with reference to the mean arterial blood pressure (mABP) at the level of the external auditory meatus. Two time points were selected (120 s apart) during which the $\mathrm{mABP}$ at the level of the brain was stable and within $5 \mathrm{mmHg}$ in the supine position and the BCP before surgery. (a) The mABP at the level of the heart; (b) the cardiac index; (c) the stroke volume index; and (d) the heart rate. Error bar chart values are expressed as the mean \pm standard deviation. ${ }^{\star} P<0.029$ in comparison to the value in the supine position. NS: not significant. White circles: non-hypertensive patients. Black circles: hypertensive patients.

in the BCP were significantly higher than those recorded while patients were in the supine position $(P<0.0001)$. The position-related changes in the CI, SVI and heart rate are shown in Figures 1 (b)-(d), respectively. The CI changed from 2.2 \pm 0.4 to $2.5 \pm 0.5 \mathrm{l} / \mathrm{min} / \mathrm{m}^{2}(P=0.13$, Figure $1(\mathrm{~b}))$ and while this value increased in nine patients, it decreased in the other four. Overall, the SVI showed a significant increase from $35.8 \pm 4.8$ to $42.3 \pm 7.8 \mathrm{ml} / \mathrm{m}^{2}(P=0.003$, Figure $1(\mathrm{c}))$. The SVI decreased in three patients and these patients also experienced a decreased CI. The heart rate changed from $63.0 \pm 10.0$ to $58.6 \pm 6.1$ beats $/ \mathrm{min}(P=0.046$, Figure $1(\mathrm{~d}))$. The SVV, which was $12.4 \% \pm 3.5 \%$ in the supine position, was significantly decreased in the BCP $(8.8 \% \pm 2.3 \%, P=0.024)$.

\section{Discussion}

In the present study, we found that when the mABP at the brain level was kept 
the same in the supine position and the BCP, then the SVI increased leaving the $\mathrm{CI}$ unaffected. Previous studies have reported that postural changes in anesthetized patients result in cardiovascular changes as the patient's head is elevated from a supine position. The elevation of the head was shown to result in a decrease in the CI [14] [15] [16] and SVI [14] [16]. Many studies have reported that the $\mathrm{mABP}$ recorded at the level of the heart decreases as patients are shifted to the BCP [8] [10] [14] [15]. However, the changes that occur in these parameters after the postural change and the increase of the BP are unknown. Similarly to previous reports, it was hypothesized that the $\mathrm{mABP}$ recorded at the level of the heart, as well as the CI, and SVI would decrease in our patients following a shift to the BCP. It was hypothesized that both the CI and the SVI (especially the SVI) would increase after the $\mathrm{BP}$ was increased in the $\mathrm{BCP}$ in comparison to the values measured in the BCP when the BP was not increased. However, the changes in the CI, SVI and heart rate showed various patterns in the present study. The main cause of this discrepancy is the various methods that were used to increase the BP. Furthermore, various methods are used to increase the BP in the clinical setting and these methods exert varying degrees of influence on the systemic circulation, including the parameters that were reported in the present study. In any case, we need to pay attention to the changes in the cardiac function and to manage patients according to their individual conditions.

The present study is associated with several limitations, namely the small sample size and the various methods that were applied to increase the BP. It is very difficult to keep the $\mathrm{mABP}$ at the level of the brain the same in both the supine position and the BCP. Thus, we could not collect a sufficient number of patients and could not use a single method to increase the BP in all patients. Our study protocol was designed to make comparisons within individuals. We calculated that a study population of 25 would be needed. Because the statistical power was $80 \%$, a difference in $\mathrm{mABP}$ of $10 \mathrm{mmHg}$ was considered to be clinically relevant, and the SD in the change in the $\mathrm{mABP}$ (between before and after the change in position) was $5 \mathrm{mmHg}$. We performed an interim analysis using the Pocock method with a study population that was half the size of that in the protocol and set the $p$ value at 0.029 instead of 0.05 in order to avoid increasing the chance of a type 1 error [13]. The methods that we used to increase the BP have been utilized in previous studies [9] [17]. However, these methods have different effects on the cardiac function. Further studies should be performed to investigate the effects of each of these methods on the cardiac function in the BCP and methods for increasing the BP should be selected according to their effects on the cardiac function.

The BCP is associated with rare but severe neurological complications [2] [3]. The cause of neurological complications in the BCP is cerebral desaturation due to the gravitational effects of the elevation of the head [6] [8] [9]. The non-invasive measurement of the $\mathrm{mABP}$ in the upper extremity will overestimate the $\mathrm{mABP}$ in the brain. In the present study, the distance between the brain and the heart was approximately $21 \mathrm{~cm}$. The $\mathrm{mABP}$ at the level of the brain was 
approximately $67 \mathrm{mmHg}$. It was calculated that the $\mathrm{mABP}$ at the level of the brain would decrease by $0.77 \mathrm{mmHg}$ for each $1 \mathrm{~cm}$ in head elevation with a heart-level $\mathrm{mABP}$ of approximately $82 \mathrm{mmHg}$. This calculated value was the same as the value that was directly recorded in the radial artery in our study (approximately $81 \mathrm{mmHg}$ ). In a conscious, healthy individuals, the cerebral blood flow is maintained at a constant level of between 50 (recently 70 has been proposed) and $150 \mathrm{mmHg}$, despite changes in the cerebral perfusion pressure [11] [18]. Reports indicate that cerebral autoregulation is unaffected by general anesthesia [18]. In our patient population, the $\mathrm{MABP}$ at the level of the brain was between 54 and $87 \mathrm{mmHg}$ and safe $\mathrm{mABP}$ levels could be maintained.

\section{Conclusion}

In conclusion, it is important to maintain the cerebral blood flow when a patient is in the BCP. However, to ensure patient safety, close attention should be paid to the systemic cardiovascular changes that occur in each patient when the BP is increased. Further studies are needed to determine optimal method for maintaining the BP that avoids both cerebral desaturation and the over-loading to the heart in anesthetized patients in the BCP.

\section{References}

[1] Peruto, C.M., Ciccotti, M.G. and Cohen, S.B. (2009) Shoulder Arthroscopy Positioning: Lateral Decubitus versus Beach Chair. Arthroscopy, 25, 891-896. https://doi.org/10.1016/j.arthro.2008.10.003

[2] Bhatti, M.T. and Enneking, F.K. (2003) Visual Loss and Ophtalmoplegia after Shoulder Surgery. Anesthesia \& Analgesia, 96, 899-902. https://doi.org/10.1213/01.ANE.0000047272.31849.F9

[3] Pohl, A. and Cullen, D.J. (2005) Cerebral Ischemia during Shoulder Surgery in the Upright Position: A Case Series. Journal of Clinical Anesthesia, 17, 463-469. https://doi.org/10.1016/j.jclinane.2004.09.012

[4] The Official Journal of the Anesthesia Patient Safety Foundation. (2009) APSF Newsletter, 24, 45-48.

[5] Murphy, G.S., Szokol, J.W., Avram, M.J., Greenberg, S.B., Shear, T.D., Vender, J.S., Levin, S.D., Koh, J.L., Parikh, K.N. and Patel, S.S. (2014) Effect of Ventilation on Cerebral Oxygenation in Patients Undergoing Surgery in the Beach Chair Position: A Randomized Controlled Trial. British Journal of Anaesthsia, 113, 618-627. https://doi.org/10.1093/bja/aeu109

[6] Hinghofer-Szalkay, H. (2011) Gravity, the Hydrostatic Indifference Concept and the Cardiovascular System. European Journal of Applied Physiology, 111, 163-174. https://doi.org/10.1007/s00421-010-1646-9

[7] ter Laan, M., van Dijk, J.M., Elting, J.W., Staal, M.J. and Absalom, A.R. (2013) Sympathetic Regulation of Cerebral Blood Flow in Humans: A Review. British Journal of Anaesthesia, 111, 361-367. https://doi.org/10.1093/bja/aet122

[8] Closhen, D., Berres, M., Werner, C., Engelhard, K. and Schramm, P. (2013) Influence of Beach Chair Position on Cerebral Oxygen Saturation: A Comparison of INVOS and FORE-SIGHT Cerebral Oximeter. Journal of Neurosurgical Anesthesiology, 25, 414-419. https://doi.org/10.1097/ANA.0b013e3182973349 
[9] Meex, I., Vundelinckx, J., Buyse, K., Deburggraeve, F., De Naeyer, S., Desollvere, V., Anné, L., Truijen, J., Vander Laenen, M., Heylen, R., De Deyne, C. and Jans, F. (2016) Cerebral Tissue Oxygen Saturation Values in Volunteers and Patients in the Lateral Decubitus and Beach Chair Positions: A Prospective Observational Study. Canadian Journal Anesthesia, 63, 537-543. https://doi.org/10.1007/s12630-016-0604-3

[10] Koh, J.L., Levin, S.D., Chehab, E.L. and Murohy, G.S. (2013) Neer Award 2012: Cerebral Oxygenation in the Beach Chair Position: A Prospective Study on the Effect of General Anesthesia Compared with Regional Anesthesia and Sedation. Journal of Shoulder and Elbow Surgery, 22, 1325-1331.

https://doi.org/10.1016/j.jse.2013.01.035

[11] Murphy, G.S. and Szokol, J.W. (2011) Blood Pressure Management during Beach Chair Position Shoulder Surgery: What Do We Know? Canadian Journal of Anesthesia, 58, 977-982. https://doi.org/10.1007/s12630-011-9573-8

[12] Ozzeybek, D., Oztekin, S., Mavioğlu, O., Karaege, G., Ozkardeşler, S., Ozkan, M., Canyilmaz, M. and Elar, Z. (2003) Comparison of the Haemodynamic Effects of Interscalene Block Combined with General Anaesthesia and Interscalene Block alone for Shoulder Surgery. Journal of International Medical Research, 31, 428-433. https://doi.org/10.1177/147323000303100512

[13] Schulz, K.F. and Grimes, D.A. (2005) Multiplicity in Randomized Trials II: SUBGroup and Interim Analyses. Lancet, 365, 1657-1661. https://doi.org/10.1016/S0140-6736(05)66516-6

[14] Buhre, W., Weyland, A., Buhre, K., Kazmaier, S., Mursch, K., Schmidt, M., Sydow, M. and Sonntag, H. (2000) Effects of the Sitting Position on the Distribution of Blood Volume in Patients Undergoing Neurosurgical Procedures. British Journal of Anaesthesia, 84, 354-357. https://doi.org/10.1093/oxfordjournals.bja.a013439

[15] Soeding, P.F., Hoy, S., Hoy, G., Evans, M. and Royse, C.F. (2013) Effect of Phenylephrine on the Haemodynamic State and Cerebral Oxygen Saturation during Anaesthesia in the Upright Position. British Journal of Anaesthesia, 111, 229-234. https://doi.org/10.1093/bja/aet024

[16] Jo, Y.Y., Jung, W.S., Kim, H.S., Chang, Y.J. and Kwak, H.J. (2014) Prediction of Hypotension in the Beach Chair Position during Shoulder Arthroscopy Using Pre-Operative Hemodynamic Variables. Journal of Clinical Monitoring and Computing, 28, 173-178. https://doi.org/10.1007/s10877-013-9512-Z

[17] Murphy, G.S., Szokol, J.W., Marymont, J.H., Greenberg, S.B., Avram, M.J., Vender, J.S., Vaughn, J. and Nisman, M. (2010) Cerebral Oxygen Desaturation Events Assessed by Near-Infrared Spectroscopy during Shoulder Arthroscopy in the Beach Chair and Lateral Decubitus Positions. Anesthesia \& Analgesia, 111, 496-505. https://doi.org/10.1213/ANE.0b013e3181e33bd9

[18] Dagal, A. and Lam, A.M. (2009) Cerebral Autoregulation and Anesthesia. Current Opinion in Anaesthesiology, 22, 547-552.

https://doi.org/10.1097/ACO.0b013e32833020be 